\title{
A Synthetic Solution for Identification and Extraction of the Effective Microseismic Wave Component Using Decomposition on Time, Frequency, and Wavelet Coefficient Domains
}

\author{
Mingwei Zhang, ${ }^{1}$ Qingbin Meng, ${ }^{1}$ Shengdong Liu, ${ }^{1}$ and Hideki Shimada ${ }^{2}$ \\ ${ }^{1}$ State Key Laboratory for Geomechanics and Deep Underground Engineering, School of Mechanics and Civil Engineering, \\ China University of Mining and Technology, Xuzhou, Jiangsu 221116, China \\ ${ }^{2}$ Department of Earth Resources Engineering, Faculty of Engineering, Kyushu University, 744 Motooka, Nishi-ku, \\ Fukuoka 819-0395, Japan
}

Correspondence should be addressed to Mingwei Zhang; mingweizhang@cumt.edu.cn and Shengdong Liu; vipshengdongliu@sina.com

Received 26 April 2017; Accepted 24 October 2017; Published 19 November 2017

Academic Editor: Juan C. G. Prada

Copyright (C) 2017 Mingwei Zhang et al. This is an open access article distributed under the Creative Commons Attribution License, which permits unrestricted use, distribution, and reproduction in any medium, provided the original work is properly cited.

\begin{abstract}
To reduce noise components from original microseismic waves, a comprehensive fine signal processing approach using the integrated decomposition analysis of the wave duration, frequency spectrum, and wavelet coefficient domain was developed and implemented. Distribution regularities of the wave component and redundant noise on the frequency spectrum and the wavelet coefficient domain were first expounded. The frequency threshold and wavelet coefficient threshold were determined for the identification and extraction of the effective wave component. The frequency components between the reconstructed microseismic wave and the original measuring signal were compared. The noise elimination effect via the scale-changed domain decomposition was evaluated. Interaction between the frequency threshold and the wavelet coefficient threshold in the time domain was discussed. The findings reveal that tri-domain decomposition analysis achieves the precise identification and extraction of the effective microseismic wave component and improves the reliability of waves by eliminating the redundant noise. The frequency threshold and the wavelet coefficient threshold on a specific time window are two critical parameters that determine the degree of precision for the identification of the extracted wave component. This research involves development of the proposed integrated domain decomposition method and provides a diverse view on the fine processing of the microseismic signal.
\end{abstract}

\section{Introduction}

In deep underground mining, microseismic events are ubiquitous. As a direct manifestation of mining-induced tremors, microseismic events are usually considered in the management of major geological dynamic hazards, for example, rock bursts or coal and gas outbursts. In addition, microseismic events play a significant role in the assessment, prediction, prevention, and control of deep dynamic disasters. Currently, for most of the research studies on microseismic activity, determining how to extract valuable information from microseismic events to represent the physical and dynamic characteristics of coal and rock masses is one of the main research interests [1-6]. These research studies focus closely on microseismic data and more specifically on the microseismic wave $[7,8]$. A microseismic wave induced from a microseismic event is acquired by advanced geophysical monitoring equipment. It is a typical complicated nonstationary signal with some unique characteristics, such as short duration, sudden saltation, and rapid attenuation $[9,10]$. Effective microseismic waves are quite necessary in mining to describe and respond to the potential characteristics in spectral analysis related to time, frequency, and energy, which are prerequisites for further studies on hazard management [11-13].

In most cases, a microseismic wave monitored in deep rock is inevitably mixed with the redundant noise, which is generally composed of firmware noise and environmental 
noise. Regarding the influence of hardware performance, the logical error of a microseismic wave itself is irrevocable. Advanced devices only help to acquire a wave as natural, original, and close to reality as much as possible by suppressing firmware noise on the premise of maintaining wave integrity. Because of signal interference from the geomagnetic field, electric discharge, and machinery operation, environmental noise actually negatively affects a microseismic wave as a result of the indeterminacy caused by unpredictable magnitude and duration $[14,15]$. The redundant noise component leads to many potential negative effects. For example, the accuracy of focus positioning and the reliability of focus energy calculation both decrease because of the vague arrival time, and an evaluation error occurs in dynamic spectral analysis because of abnormal frequencies. In particular, noise interference results in tiny differences between the real wave and the monitored wave; these differences decrease the accuracy and reliability of characterizing a microseismic wave $[16,17]$. Thus, adequate attention must be paid to the noise elimination effect of a microseismic wave.

Noise in a signal is impossible to eliminate completely because of the superposition of the redundant noise frequency and the effective wave frequency $[18,19]$. Regarding frequency superposition, two challenges must be faced. One challenge is the range determination of the frequency distribution of the redundant noise and the effective wave in the entire spectrum; another challenge is the proportion confirmation of the frequency magnitude of the redundant noise and the effective wave on the same frequency band. Determining how to reduce the redundant noise component from the original signal as much as possible has always been a difficult problem to solve. In general signal processing, for example, via use of a low-pass filter and wavelet denoising, although these approaches are developed and have contributed to many achievements, the noise elimination effect of such approaches on the original microseismic signal is unstable and imprecise. Much of the effective wave component is lost with the noise reduction. In addition, noise is still abundant in the reserved portion of the signal. Noise in the original microseismic signal is passive. Noise frequencies are abundant in the entire frequency domain; that is, it is not regional but mixed thoroughly with the effective wave frequency, which is the intrinsic attribute of the microseismic signal. Because of the abundance of the noise component and the uncertainty of the noise distribution in the entire frequency domain of the original microseismic signal, the noise elimination capacity of each approach is limited. Limitation and applicability of the general denoising methods play negative roles on the noise elimination effect. The adaptability of each method is different for distinct signals. Noise reduction cannot be completely solved using only one approach.

Microseismic wave reliability will be improved significantly as the redundant noise component is reduced as much as possible; in other words, the effective wave component is extracted completely. Thus, in this study, the authors integrate the advantages of the low-pass filter and wavelet denoising and introduce a fine noise elimination method based on the scale-changed domain decomposition on wave duration, frequency spectrum, and wavelet coefficient. By extracting the real wave component from the original monitored signal, the noise component is reduced as much as possible, and the high-quality microseismic wave component is obtained. This approach is applicable for a microseismic wave with an abundant noise component. Current research studies on the precise identification and extraction of the effective microseismic wave component are rarely achievable and appear to be quite scarce and limited on microseismic wave processing [20-22]. Here, we examine how it can be more effective in achieving the noise elimination effect using wave component identification and extraction. Moreover, this study emphasizes the necessity for improving the reliability of processing a microseismic wave and provides a diverse research view for the basic processing of microseismic waves.

\section{Principle of Scale-Changed Tri-Domain Decomposition Analysis}

Precise identification and extraction of the effective wave component are one form of fine wave processing. Domain decomposition analysis is a comprehensive approach for implementing effective component identification and extraction via the conjoint wave decomposition in the time domain, frequency domain, and wavelet coefficient domain. For a fixed length of wave, the effective component of a subwave with a specific time window is extracted upon a few threshold frequencies in a subband. A preliminary effective wave is then reconstructed based on the wavelet coefficients of the processed subwaves. Immediately following the preliminary extraction, advanced extraction to obtain the effective wave component is conducted for subwaves with the specific threshold frequency using a few threshold coefficients; a relatively pure wave is finally achieved after wavelet packet reconstruction of the effective wave component. The main purpose of advanced extraction is separation of the high frequency low-energy redundant component from the original wave to leave only the low frequency high-energy effective component. An analytical model for the scalechanged tri-domain decomposition analysis is illustrated in Figure 1.

Fine wave decomposition is conducted synthetically in the time domain, frequency domain, and wavelet coefficient domain. The precision of the decomposition depends on the complexity of the wave structure, which is worthy of special consideration in effective component extraction. In most cases, such as the noise randomization, the mixed redundant component cannot be confirmed quantitatively, and the real microseismic wave is unknowable; as a result, the integral microseismic wave is relatively complicated compared to conventional signals. When using domain decomposition analysis, one important issue is reasonable determination of the threshold frequency and the threshold coefficient. The threshold frequency and the threshold coefficient used for one wave are usually different from those for others. Only as these two parameters are reasonably determined can effective wave component extraction be possibly achieved. 


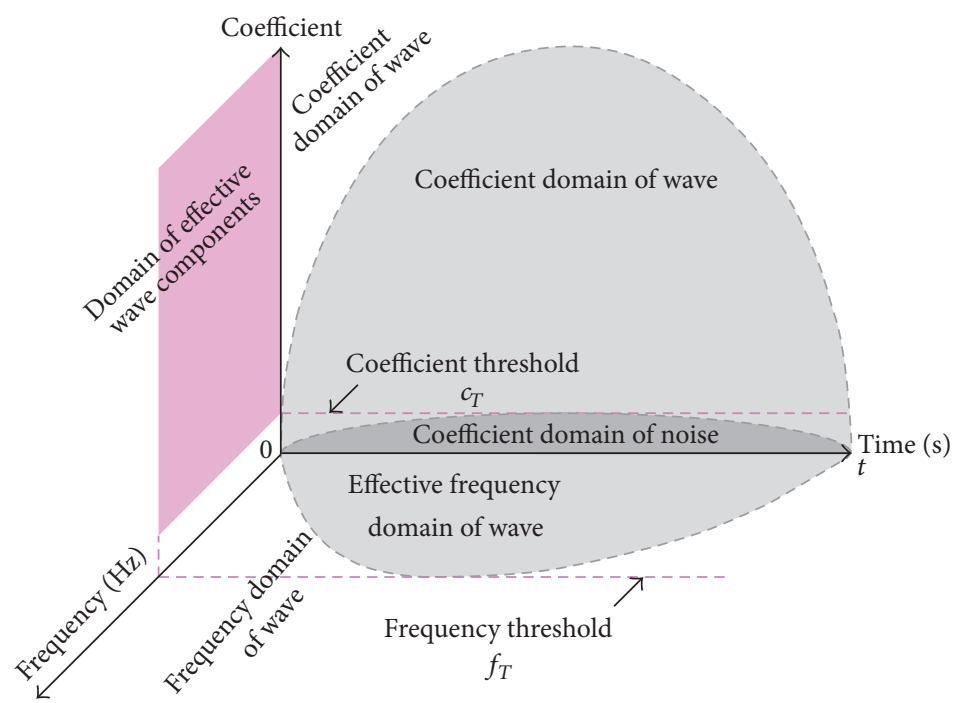

FIGURE 1: Schematic of the analytical model of the scale-changed domain decomposition analysis. The time domain is determined by the time duration; the thresholds in the frequency domain and the wavelet coefficient domain are restricted by the effective wave component and the peak noise coefficient, respectively.

2.1. Wavelet Packet Function and Transform. In the wavelet multiresolution analysis, the Hilbert space is decomposed as the orthogonal sum of all wavelet subspaces $W_{j}(j \in Z)$, which is a closure of the wavelet function according to the different scales [23]. To improve the frequency resolution, the wavelet subspace is decomposed based on the binary mode $[24,25]$. In Hilbert space, scale subspace $V_{j}$ and wavelet subspace $W_{j}$ are represented by a unified space $U_{j}$; that is,

$$
\begin{gathered}
V_{j+1}=V_{j}+W_{j}, \\
U_{j}^{0}=V_{j}, \\
U_{j}^{1}=W_{j}, \\
\Downarrow \\
U_{j+1}^{0}=U_{j}^{0}+U_{j}^{1} .
\end{gathered}
$$

If $U_{j}^{n}$ and $U_{j}^{2 n}$ are the closure spaces of function $u_{n}(t)$ and $u_{2 n}(t)$, respectively, and satisfy the following dual-scale equation:

$$
\begin{aligned}
u_{2 n}(t) & =\sqrt{2} \sum h(k) u_{n}(2 t-k), \\
u_{2 n+1}(t) & =\sqrt{2} \sum g(k) u_{n}(2 t-k),
\end{aligned}
$$

$$
k \in Z, n=1,2,3, \ldots
$$

then

$$
U_{j+1}^{n}=U_{j}^{2 n}+U_{j}^{2 n+1} .
$$

The orthogonal wavelet packet decided by base function $u_{0}(t)$ is then defined as

$$
\begin{aligned}
W_{j}= & U_{j-k}^{2^{k}}+U_{j-k}^{2^{k}+1}+\cdots+U_{j-k}^{2^{k}+m}+\cdots+U_{j-k}^{2^{k+1}-2} \\
& +U_{j-k}^{2^{k+1}-1} .
\end{aligned}
$$

2.2. Frequency Decomposition by the Wavelet Packet Transform. A wavelet packet function can be briefly expressed as $\varphi_{j, k, n}(t)\left(n=2^{k}+m\right)$, in which parameters $j, k$, and $n$ represent the scale, displacement, and frequency indicator, respectively [26]. As the frequency representation, parameter $n$ overcomes a defect of poor resolution in the high frequency range. Using the wavelet packet transform, $2^{n}$ frequency subbands are obtained at the $n$th decomposition layer. The bandwidth of each frequency subband is $f_{\max } / 2^{n}$ ( $f_{\max }$ is the maximum frequency). The wave component in separate frequency bands is clearly identified in detail by the deep decomposition. Moreover, original wave $s(t)$ can be completely reconstructed by these aequilate subbands [27, 28]. Figure 2 shows the wavelet packet decomposition at the fourth level.

2.3. Wave Energy Representation by the Wavelet Packet Coefficient. Wavelet packet decomposition at a specific level $i$ can be defined as [29]

$$
s(t)=\sum_{j=0}^{2^{i}-1} f_{i, j}\left(t_{j}\right)
$$

where $f_{i, j}\left(t_{j}\right)$ is the reconstructed component in both decomposition level $i$ and frequency bandwidth $f_{\max } / 2^{i}$. According to the Parseval theorem, finite energy of a random wave in each frequency band is calculated by $[30,31]$

$$
E_{i, j}=\int_{T}\left|f_{i, j}\left(t_{j}\right)\right|^{2} d t=\sum_{k=1}^{N}\left|x_{j, k}\right|^{2},
$$

where $x_{j, k}\left(j=0,1,2, \ldots, 2^{i}-1\right)$ is the amplitude of the discrete points of reconstructed wave $f_{i, j}\left(t_{j}\right)$ and $N$ is the 


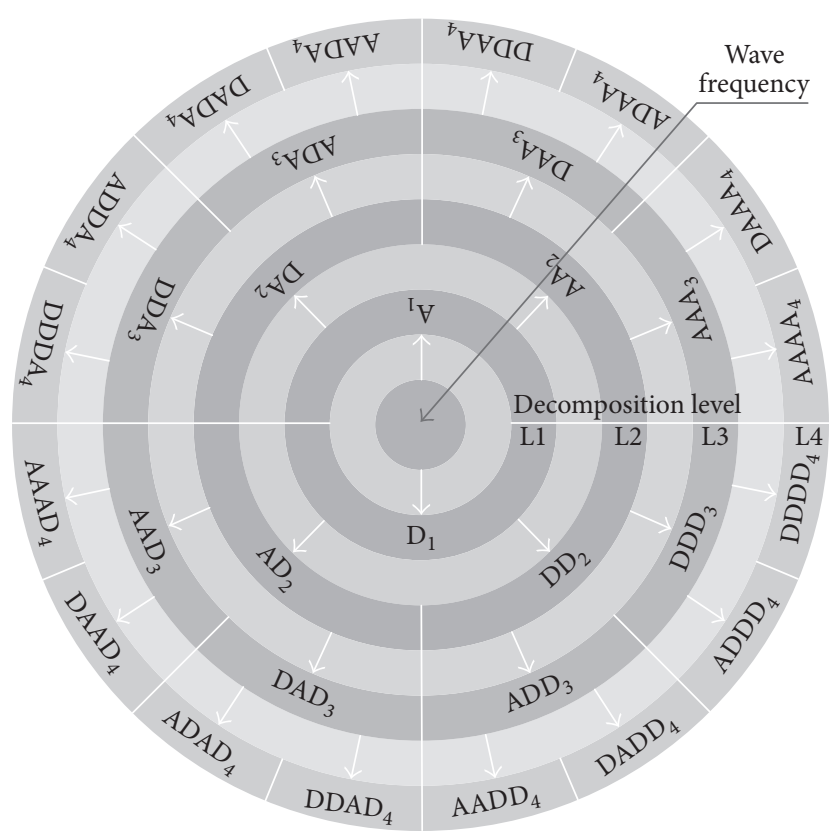

FIGURE 2: Decomposition structure in the frequency domain of a wave via the wavelet packet transform. The original wave at the basic level passes through two complementary filters (low-pass and high-pass) and emerges as two subwaves $A_{1}$ and $D_{1}$. Subwave decomposition at the sublevel follows the same rules.

number of wave points. The total wave energy is then defined as

$$
E_{w}=\sum_{j=0}^{2^{i}-1} E_{i, j}
$$

\section{Identification and Extraction of the Microseismic Wave Component Using Scale-Changed Tri-Domain Decomposition}

To specify how to achieve the identification and extraction of the microseismic wave component from the original signals as effectively as possible using the tri-domain decomposition, a microseismic wave group obtained from the Xingcun underground coal mine, located in East China with a focal mining depth of -893 meters, was selected randomly from a huge database and treated as the study object. The microseismic signal was measured using the Seismological Observation System, which was developed by the Poland Coal Research Institute and has been widely applied in many coal mines around the world. The basic function of the system is monitoring the seismic events by determining their focus location and then calculating their energy release. The sampling frequency, recording dynamic range, nonlinear error, and signal transmission form used in this field investigation were $500 \mathrm{~Hz}, \leq 110 \mathrm{~dB},<3 \%$, and current mode, respectively. The key components and a schematic diagram of the wave monitoring system are shown in Figure 3.

Original waveforms and basic characteristic information of the microseismic wave case, for example, the arrival time of the longitudinal wave ( $\mathrm{P}$ wave), the arrival time of the transverse wave (S wave) and the peak particle velocity (PPV), are shown in Figure 4.

3.1. Characteristics of Noise in the Microseismic Wave Group. No microseismic wave can remain pure during propagation in a rock medium. All of the waves measured in different locations are affected by various forms of noise. The differences are merely the interference degree on the wave propagation path. The waveform appears pure and smooth when the noise level is low. In contrast, a high noise level results in a rough waveform. Firmware noise is always present; changes are due to the environmental noise, which actually plays a more negative role. The noise component in the original wave is relatively low for close waves, whereas they become significant with the increase in the propagation distance. Noise is unknown, quite irregular, and difficult to identify visually. The noise component and magnitude in a measured microseismic wave cannot be determined because noise is natural and random; as a result, after the denoising process, whether the abandoned noise component is the objective noise or not is difficult to determine. Visual comparison cannot be used to distinguish between real noise and discarded noise, and the reliability of the denoising effect is difficult to evaluate.

For the original wave case, the amplitude in each channel is diverse. Noise is seriously suppressed by the strong microseismic wave and is quite inconspicuous in those individual waves, such as sw1, sw2, sw3, sw6, and sw7. Noise only appears relatively obvious in waves with lower amplitudes, for example, sw5, sw8, sw9, and sw12. According to the waveform, only sw12 shows some repeated noise interference; that is, the noise component in sw12 is completely regular, and the corresponding waveform can be captured. In this case, the repeated noise can be used to achieve the objective compression with the abandoned noise component to verify the accuracy of the method applied in the research. Thus, an individual microseismic wave monitored by the twelfth detector is separated from the measured wave group and is taken as a study sample to highlight the extraction process of the effective wave component. The waveform and reference noise of the effective wave component are shown in Figure 5, from which it is found that wave sw12 is at least interfered by three types of environmental noises other than firmware noises. Its wave front and tail are obscure. The PPV of wave sw12 is $0.012 \mathrm{~mm} / \mathrm{s}$, which is almost seven times the PPV of noise. The effective component in wave sw12 is not easy to identify from the original waveform alone.

\subsection{Frequency Threshold Determination for Preliminary} Component Separation. One of the important issues when extracting the effective wave component is the determination of the threshold frequency. For a wave with a simple structure, one or two threshold frequencies shall meet the preliminary processing requirements. However, a few or more threshold frequencies may become necessary to satisfy the extraction requirements. This depends entirely on the complexity of the structural characteristics in the wave frequency domain. 


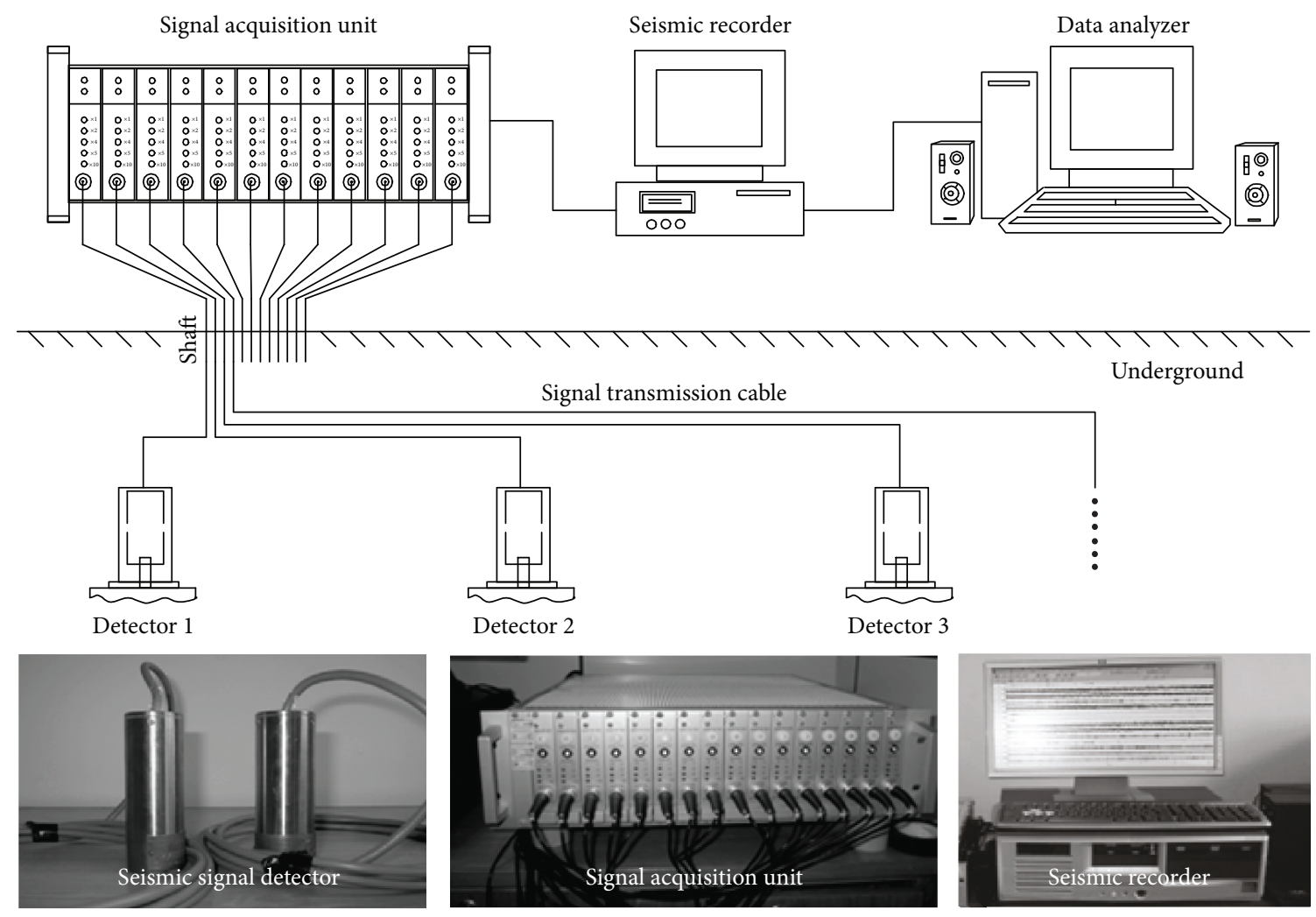

FIGURE 3: Organizational structure and key components of the applied microseismic monitoring instrument.

In most cases, the frequency of the effective microseismic wave component contributes in the low range gradually with the increase in the wave propagation distance, whereas the frequency of the redundant noise component is always distributed over the entire spectrum. Hence, a threshold frequency is set for separating the frequency domain of the effective wave only.

According to the variation of the frequency spectrum in Figure 6(a), the redundant noise component is distributed throughout the entire spectrum, with particular concentration in a specific high frequency range. Distribution of firmware noise frequency is relatively even without many obvious undulations in the entire spectrum. The average level floats around the magnitude of $3.07 \times 10^{-3}$. Firmware noise has little effect on the microseismic wave and is nearly negligible. By contrast, distribution of environmental noise frequency becomes relatively significant and exhibits an obvious undulation in the high frequency range. The average level is approximately at the magnitude of $7.02 \times$ $10^{-3}$, more than twice the levels of firmware noise. Obviously, the component of environmental noise is mainly concentrated in the high frequency domain of greater than $160 \mathrm{~Hz}$. The amplitude of the noise changes for frequencies greater than $70 \mathrm{~Hz}$ and achieves a peak of $1.05 \times 10^{-1}$ at the band at $215 \mathrm{~Hz}$. Among these three environmental sources, noise (III) is judged to be the major cause of interference. The spectral characteristics of noise frequency indicate that the redundant component in the original microseismic wave cannot be filtered out completely. Thus, extracting as much of the effective wave component as possible must be performed.

As shown in Figure 6(b), conspicuous spectral distribution of the frequency component of wave sw12 is mainly concentrated in the low range, particularly in the range of $0 \sim 41.4 \mathrm{~Hz}$. The dominant frequency is $16.848 \mathrm{~Hz}$. Moreover, an inconspicuous frequency difference appears in the range of $41.4 \sim 81.1 \mathrm{~Hz}$. It is inferred that the original measured wave shares approximately 75 percent of the appreciable frequency range with the redundant component, and the goodness of fit is quite high, especially for frequencies greater than $81.1 \mathrm{~Hz}$. The pure wave component only results in a dramatic frequency increase in the low range of $0 \sim 41.4 \mathrm{~Hz}$ and a slight increase in the frequency range of $41.4 \sim 81.1 \mathrm{~Hz}$. Thus, the frequency range of $0 \sim 81.1 \mathrm{~Hz}$ shall be considered the acceptable range of the effective wave component; that is, the band at $81.1 \mathrm{~Hz}$ can be taken as a threshold frequency for wave sw12 as the simple spectral superposition between the effective wave and the pure noise component. In addition, for the wave tail, residual wave energy only induces faint changes in the low frequency range of less than $31.25 \mathrm{~Hz}$. The band at $31.25 \mathrm{~Hz}$ thus can be taken as another threshold frequency. Frequency comparison indicates the conspicuous distribution characteristics of the wave component in the entire spectrum. The effective wave component has an extremely narrow bandwidth and is preferentially concentrated in the low frequency range; in addition, the magnitude is significantly greater than that of the redundant component in the superposed range. The evident response of the spectral 


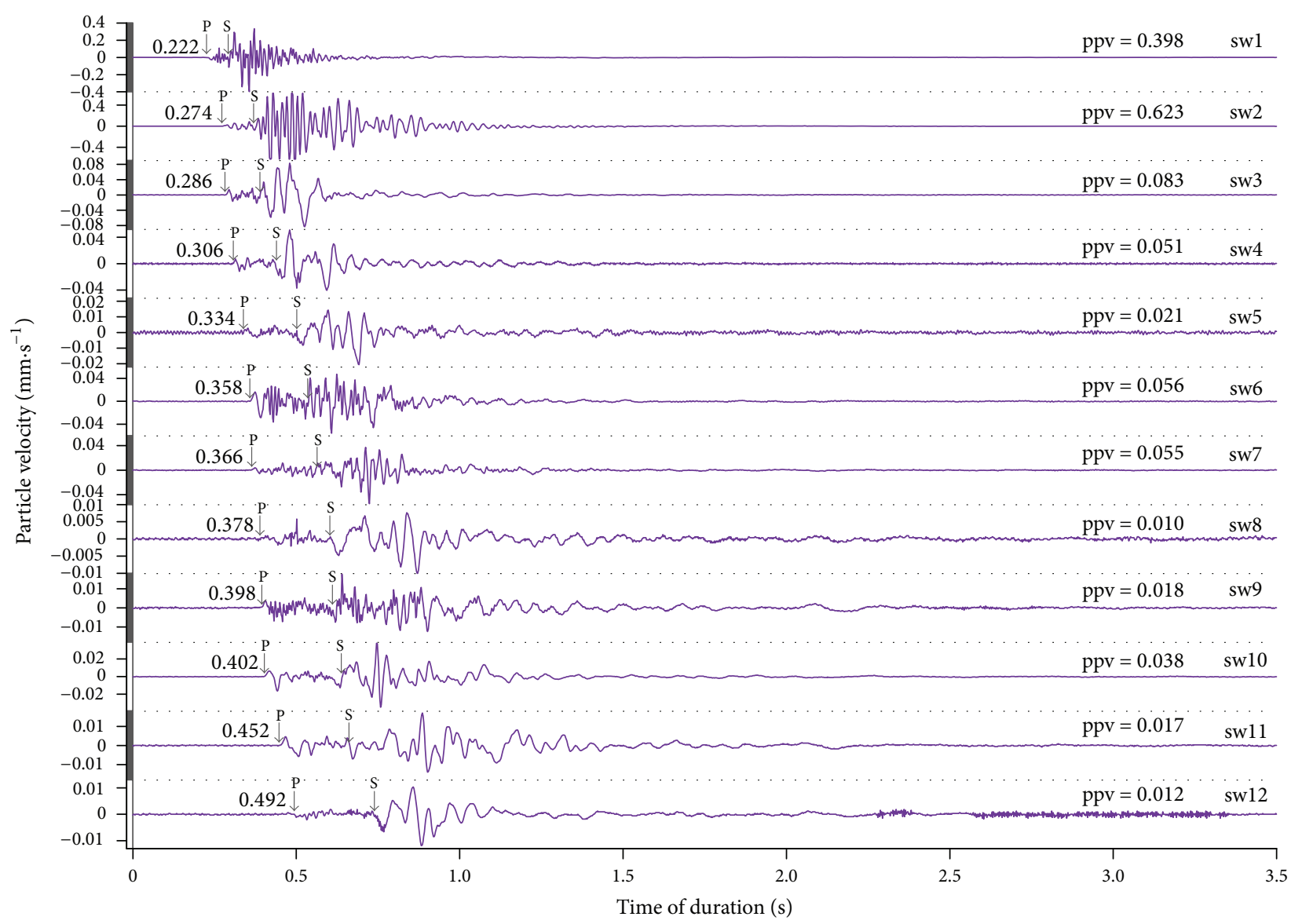

FIGURE 4: Waveform and basic characteristics of the measured microseismic wave group. Because of the impact of the velocity threshold set initially in the monitoring instrument, the PPV value of wave sw2 is not measured, and the velocity threshold limits the real particle velocity.

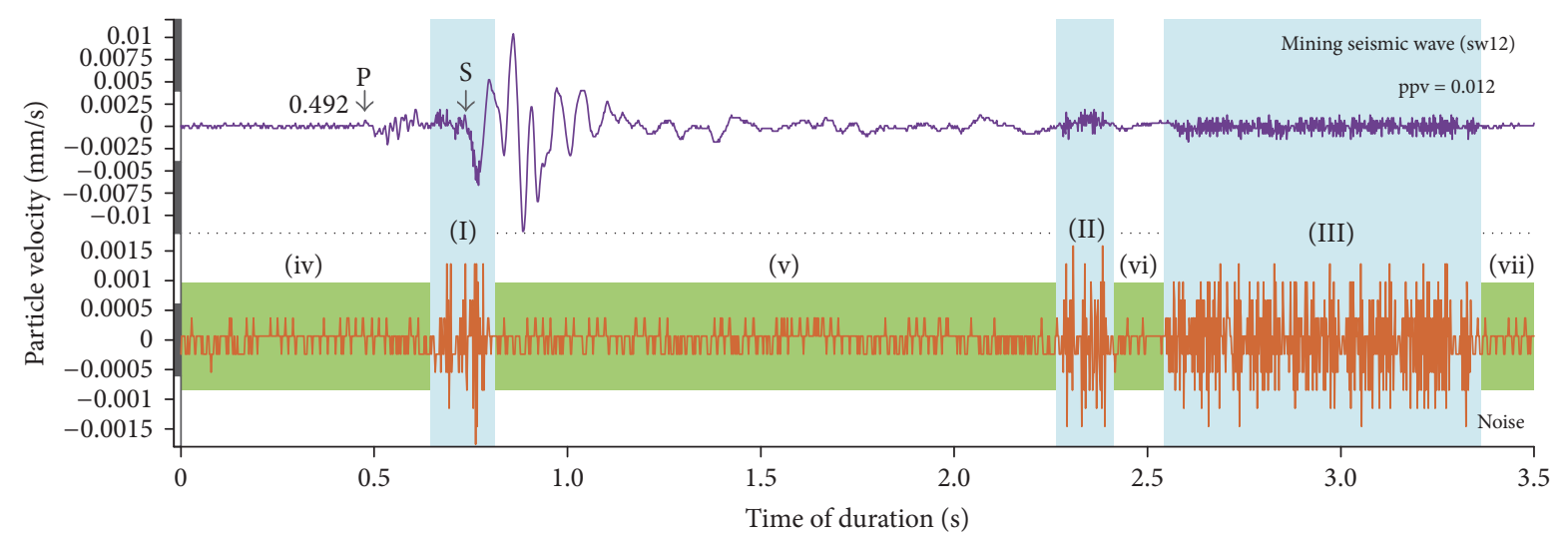

Environmental noise: (I), (II), (III)

Firmware noise: (iv), (v), (vi), (vii)

FIGURE 5: Waveforms of original microseismic wave sw12 and the reference noise.

phenomenon signifies that it is entirely feasible to extract the effective microseismic wave component from only the low frequency range.

The time-frequency characteristics of the Short Time Fourier Transform (STFT) and the Wavelet Transform are compared in Figure 7. It can be seen that the domain frequency of the microseismic wave 12 \# can be identified quickly from the imaging of these two methods. However, the STFT results are quite rough. The time resolution and the frequency resolution are difficult to improve simultaneously. In addition, the types of noise cannot be clearly determined. In contrast, the results of the Wavelet Transform are quite fine 


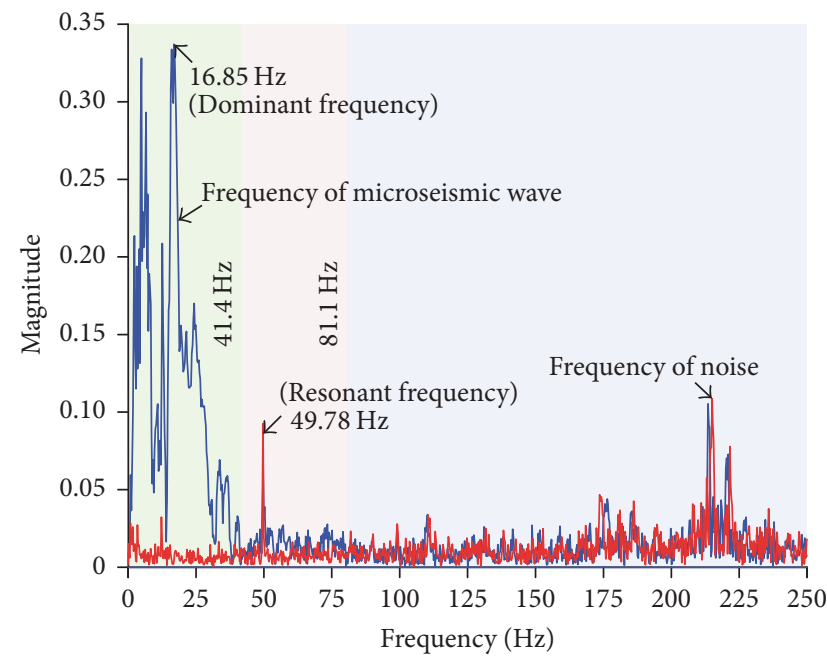

(a)

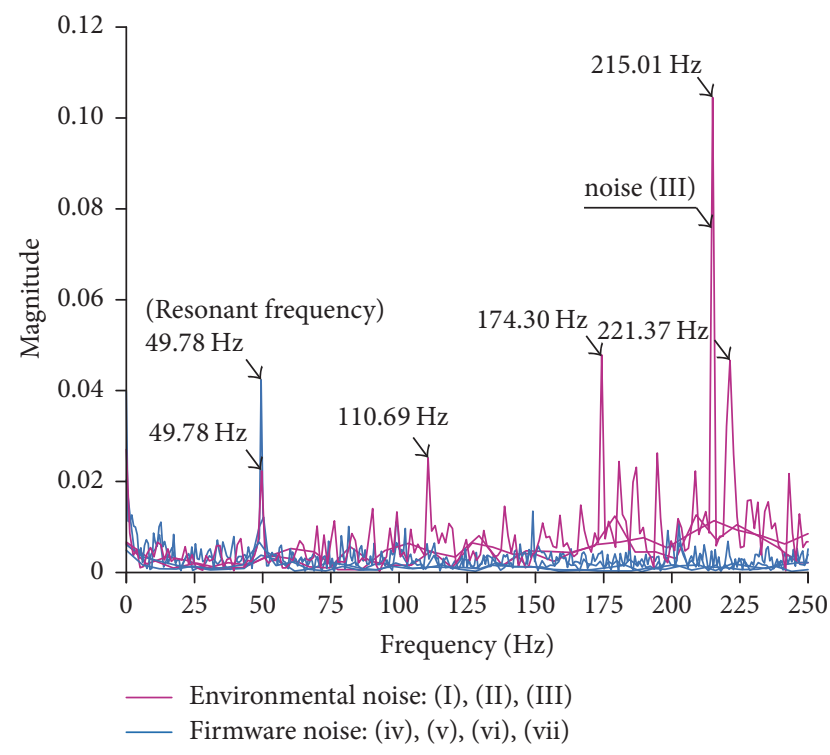

(b)

FIGURE 6: Comparison of the frequency spectra of standalone environmental noise (I, II, III) and firmware noise (iv, v, vi, vii), integrated random noise, and the measured microseismic wave.

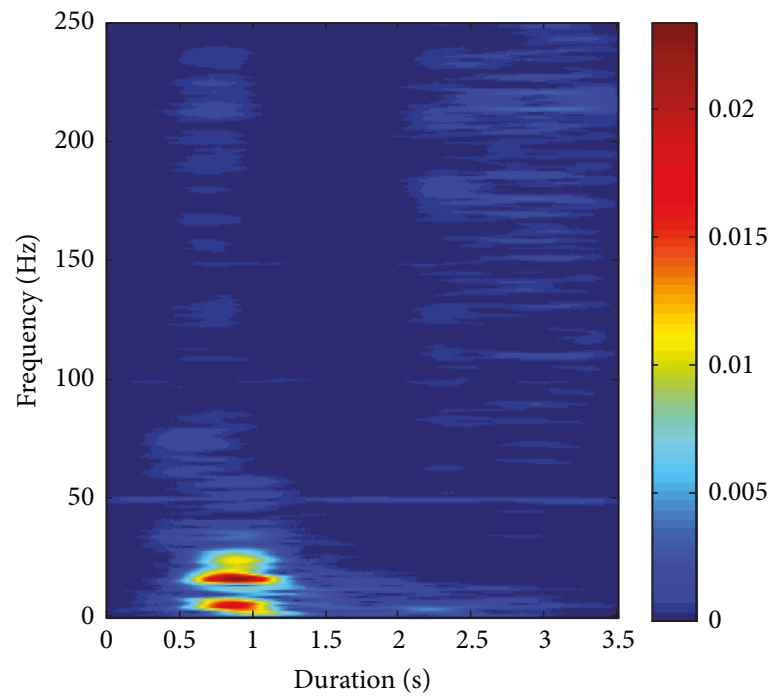

(a) STFT

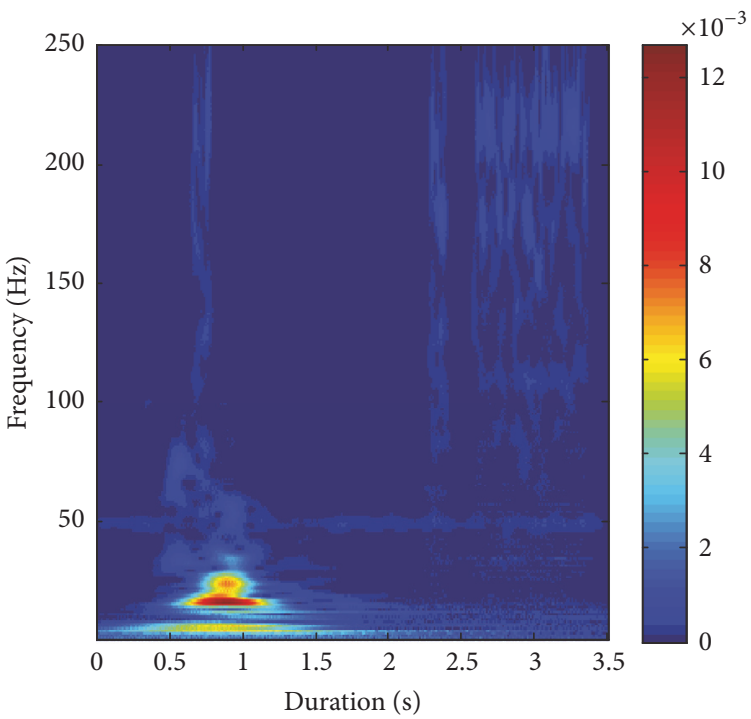

(b) Wavelet Transform

FIgURE 7: Time-frequency characteristics of the STFT and the Wavelet Transform.

and smooth. The time resolution and frequency resolution can be improved at the same time based on the requirements. The noise components in microseismic waves can be identified directly because the width of the STFT window function is changeless. The sampling interval in the time domain and the frequency domain is a constant. The size and shape of the window are independent of the frequency, and the localization of both time and frequency cannot be achieved. of the Wave Component. For extraction of the effective microseismic wave component, another key point is the identification of the threshold coefficient that is used for separating the coefficients of the pure wave and that of random noise. Extraction of the effective wave component can be achieved only if its wavelet coefficients are reserved. The wavelet coefficient threshold is the key factor in the distortion and extraction error of the effective wave and directly determines the quality of the extracted effective component. The necessary threshold coefficient is restricted by the frequency domain in a specific time domain, and its magnitude depends on the peak wavelet coefficient of the redundant component. Because of the obvious difference in 
TABLE 1: Necessary wavelet packet coefficient thresholds restricted by the frequency threshold in a specific time window.

\begin{tabular}{lccccccc}
\hline Threshold & \multicolumn{7}{c}{ Wavelet packet coefficient $\left(\times 10^{-3}\right)$} \\
(I) & (II) & (III) & (iv) & (v) & (vi) & (vii) \\
\hline Frequency (Hz) & & & & & & & \\
$0 \sim 31.25$ & $/$ & 3.72 & 3.72 & $/$ & $/$ & 2.185 & 2.185 \\
$31.25 \sim 250$ & $/$ & 1.356 & 1.356 & $/$ & $/$ & 1.219 & 1.219 \\
$0 \sim 81.1$ & 3.72 & $/$ & $/$ & 2.185 & 2.185 & $/$ & $/$ \\
$81.1 \sim 250$ & 1.356 & $/$ & $/$ & 1.219 & 1.219 & $/$ & $/$ \\
\hline
\end{tabular}

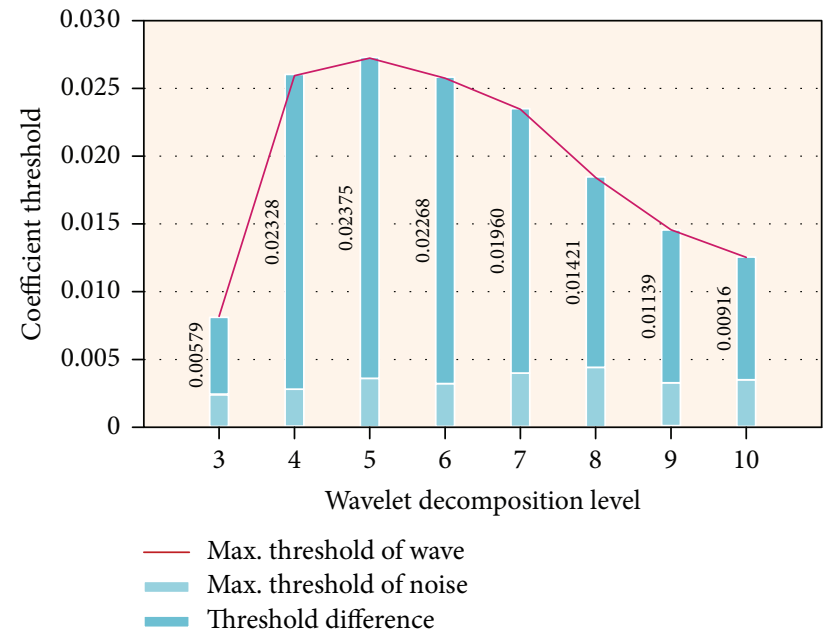

FIGURE 8: Changes in the magnitude of the maximum wavelet coefficient of the monitored microseismic wave and random noise with the decomposition level. The maximum coefficient magnitude of the monitored microseismic wave increases first from level 3 to level 5 and then decreases after level 5. The maximum coefficient magnitude of random noise continues to increase in a fluctuating manner until reaching level 8 . The microseismic wave achieves the best wavelet packet decomposition at level 5 .

noise wavelet coefficients, two or more threshold coefficients are usually necessary for precise identification of the effective microseismic wave component.

Throughout the extraction of the effective wave component, basis function sym5 is applied to the wavelet packet transform of the microseismic wave as the optimal basis [10]. As the magnitude of the wavelet coefficients is not static but changes with the decomposition level, the wavelet coefficients of the effective wave component and those of the random noise component present some distinct characteristics at different decomposing levels, as shown in Figure 8.

It is found that the difference between the maximum coefficients of the effective wave and maximum coefficients of random noise increases to the peak at the fifth level, indicating that most of the effective wave component shall be extracted when the original wave is decomposed by the basis sym 5 at level 5 . Hence, the optimum decomposition level is determined based upon the coefficient of variation. The wave component is proportional to the magnitude of the corresponding wavelet coefficients. After the reranking of the wavelet coefficients according to the rules of the Gray code, the distribution of the coefficient magnitude of the monitored microseismic wave and noise in the time-frequency conjoint domain is shown in Figure 9.

Figure 9 shows that the component of a pure wave is concentrated in a localized region of the time-frequency conjoint domain. Figure 9 synthetically represents the concentration characteristics of the wavelet coefficients in the frequency spectrum with temporal variation, indicating that the magnitude of the wavelet coefficients is the energy representation of the wave component in a time series. The coefficient magnitude distribution of the microseismic wave and random noise in the time-frequency conjoint domain implies the clear ingredient characteristics and provides a reliable guarantee of the spectral decomposition. Corresponding to the threshold frequency, the threshold coefficients used for extracting the effective wave component in each time window are confirmed by the peak wavelet coefficient of the noise component, as listed in Table 1.

\section{Results and Analysis}

4.1. Effective Component Extraction of the Microseismic Waves. The microseismic wave component is identified and extracted effectively after the above-described processing; the purified wave is shown in Figure 10. Compared to the original wave, the waveform of the extracted wave is obviously clearer. The arrival time, which changes from $0.492 \mathrm{~s}$ to $0.462 \mathrm{~s}$, is more easily recognizable and is effectively corrected by the wave extraction process. The PPV changes infinitesimally. The difference is only $0.179 \mu \mathrm{m} / \mathrm{s}$, which is a $1.72 \%$ decrease of the original PPV. The main variation lies in the frequency distribution of the extracted effective wave. Comparison of the frequency spectrum of the extracted effective wave and that of the original wave is shown in Figure 11. The frequency spectrum of the extracted effective wave is more concise. The dominant frequency changes are minor from $16.848 \mathrm{~Hz}$ to $17.133 \mathrm{~Hz}$, with the magnitude increasing slightly from 0.347 to 0.353 . Obvious changes appear in the high frequency domain. The magnitude in the frequency range greater than $110 \mathrm{~Hz}$ tends toward zero, indicating that the random noise component is almost completely absent. Insignificant frequency variation appearing in the low range indicates that the effective wave component is extracted perfectly. Finally, the original microseismic wave is purified. It turns out that the effective wave achieves an excellent extraction process from the original measured wave via the scale-changed domain decomposition. In addition, superposition of the redundant 


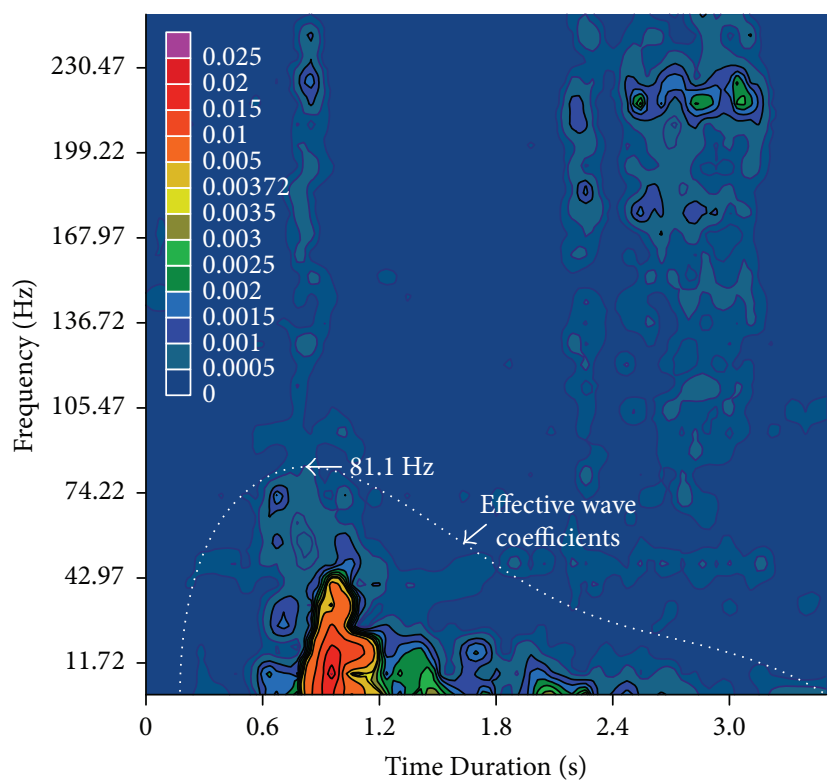

(a)

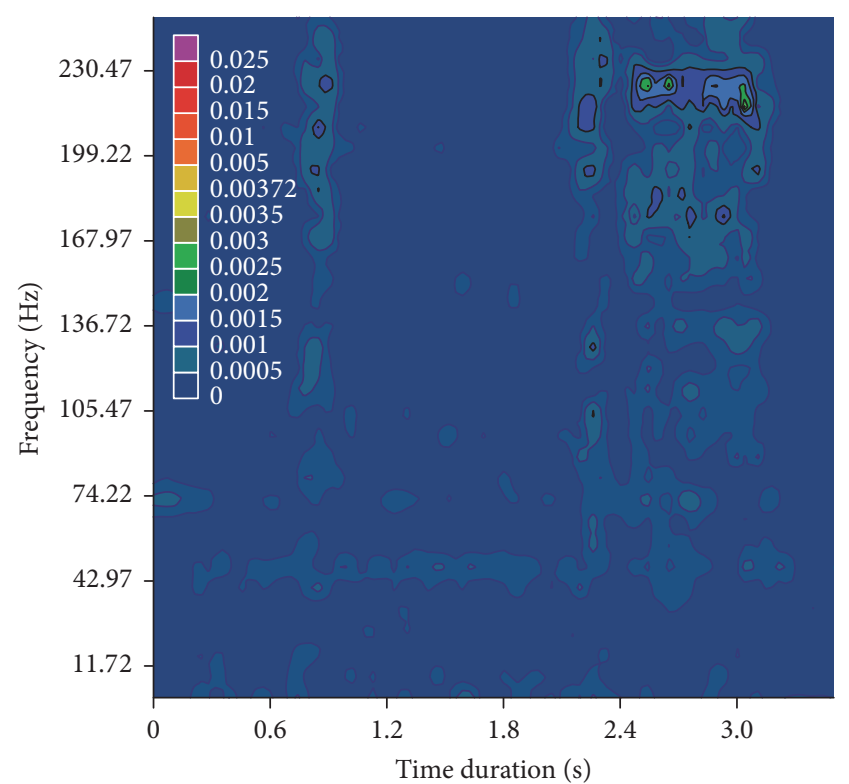

(b)

Figure 9: Coefficient magnitude distribution of the monitored microseismic wave and the reference noise in the time-frequency conjoint domain after performing the wavelet packet transform using the basis sym 5 at level 5 .

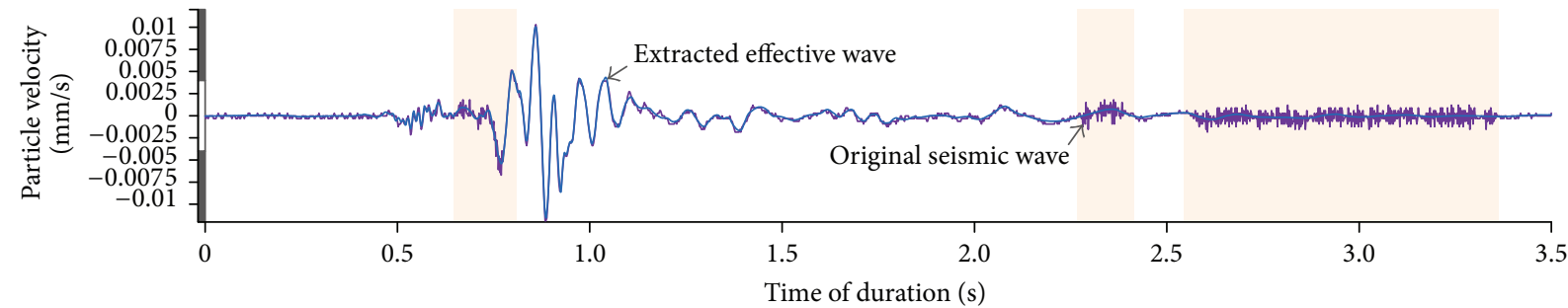

FIGURE 10: Pure microseismic waveform reconstructed via the effective wave component extracted from the original measured wave sw12.

noise frequency and the effective wave frequency is effectively addressed.

\subsection{Evaluation of the Extracting Reliability of the Effective} Wave Component. To verify the extracting reliability of the effective wave component, quantitative evaluation of the wave effect is performed based on the Signal-to-Noise Ratio (SNR) and the energy-reservation ratio $\eta_{E}$. The relevant expressions are defined as

$$
\begin{aligned}
\mathrm{SNR} & =10 \log \left(\frac{\sum_{1}^{N} \widehat{S}^{2}(i)}{\sum_{1}^{N}[S(i)-\widehat{S}(i)]^{2}}\right), \\
\eta_{E} & =\frac{\sum_{1}^{N} E_{\widehat{S}(i)}}{\sum_{1}^{N} E_{S(i)}} \times 100 \%,
\end{aligned}
$$

where $S(i)$ and $\widehat{S}(i)$ are the original microseismic wave and the extracted wave, respectively. $N$ is the wave length. High values of the SNR and the energy-reservation ratio generally ensure the well-preserved characteristics of the effective wave and well-satisfied requirements of the wave smoothness and necessarily indicate a great extraction effect. The SNR value and energy-reservation ratio of the extracted effective wave are compared with the results obtained from other conventional approaches, as listed in Table 2.

This table directly reveals the excellent wave extracting effect of the scale-changed domain decomposition, by which the effective wave components have the highest values of the SNR and the energy-reservation ratio. The reliability of the desired microseismic wave is indeed enhanced. Note that the energy of the extracted effective wave is a direct consequence of subtracting the pure noise energy from the original wave energy. Only quite small amounts of wave energy from the threshold coefficient in the low frequency range are missing.

4.3. Precise Extraction of the Effective Component for Microseismic Wave Group. Following the abovementioned extraction process for the effective wave component, the extracted results of pure microseismic waves in other monitoring channels are shown in Figure 12. Moreover, the acceptable frequency domain of the extracted effective microseismic waves in the monitoring channels and the corresponding variation of a few dominant parameters, such as the dominant frequency, wave energy, SNR, and energy-reservation ratio, are listed in Table 3. 
TABLE 2: Comparison of the SNR value and the energy-reservation ratio of the effective wave processed using diverse approaches.

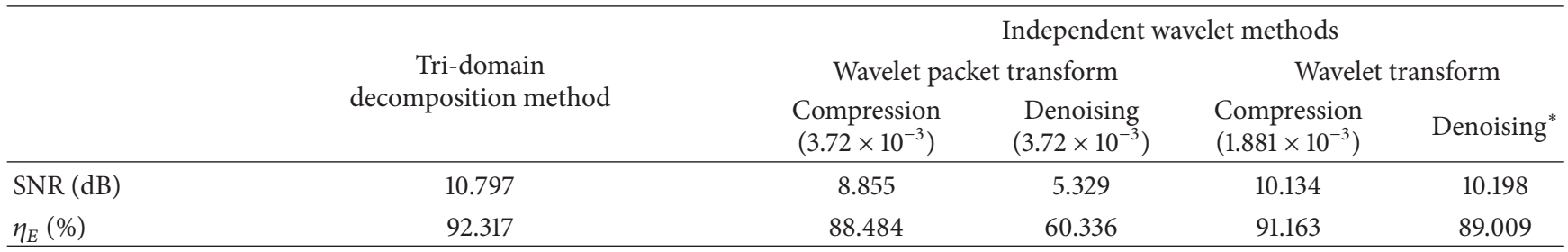

${ }^{*}$ Coefficient thresholds for the wavelet denoising at levels $1,2,3,4$, and 5 are $2.096 \times 10^{-3}, 1.258 \times 10^{-3}, 1.013 \times 10^{-3}, 0$, and 0 , respectively.

TABLE 3: Acceptable frequency domain and variation of the dominant parameters of the extracted effective microseismic waves in all of the monitoring channels.

\begin{tabular}{lccccc}
\hline Wave group & $\begin{array}{c}\text { Reserved frequency domain } \\
(\mathrm{Hz})\end{array}$ & $\begin{array}{c}\text { Dominant frequency } \\
(\mathrm{Hz})\end{array}$ & $\begin{array}{c}\text { Wave energy } \\
(\mathrm{J})\end{array}$ & $\begin{array}{c}\text { SNR } \\
(\mathrm{dB})\end{array}$ & $\begin{array}{c}\text { Energy reservation ratio } \\
(\%)\end{array}$ \\
\hline sw1 & $0 \sim 196.7$ & 93.95 & 1705.43 & 11.262 & 91.541 \\
sw2 & $0 \sim 107.5$ & 36.27 & 17948.46 & 10.728 & 88.327 \\
sw3 & $0 \sim 151.1$ & 10.85 & 162.74 & 9.782 & 93.374 \\
sw4 & $0 \sim 67.1$ & 21.70 & 47.91 & 10.692 & 90.198 \\
sw5 & $0 \sim 54.3$ & 17.70 & 8.42 & 12.014 & 86.136 \\
sw6 & $0 \sim 134.2$ & 23.42 & 78.33 & 10.962 & 91.355 \\
sw7 & $0 \sim 121.4$ & 13.42 & 32.78 & 9.488 & 92.027 \\
sw8 & $0 \sim 38.3$ & 16.85 & 2.79 & 10.112 & 88.935 \\
sw9 & $0 \sim 144.2$ & 5.71 & 6.12 & 10.771 & 93.618 \\
sw10 & $0 \sim 94.5$ & 7.42 & 26.43 & 12.236 & 86.194 \\
sw11 & $0 \sim 45.7$ & 11.71 & 9.39 & 11.543 & 91.382 \\
sw12 & $0 \sim 41.4$ & 17.13 & 3.51 & 10.797 & 92.317 \\
\hline
\end{tabular}

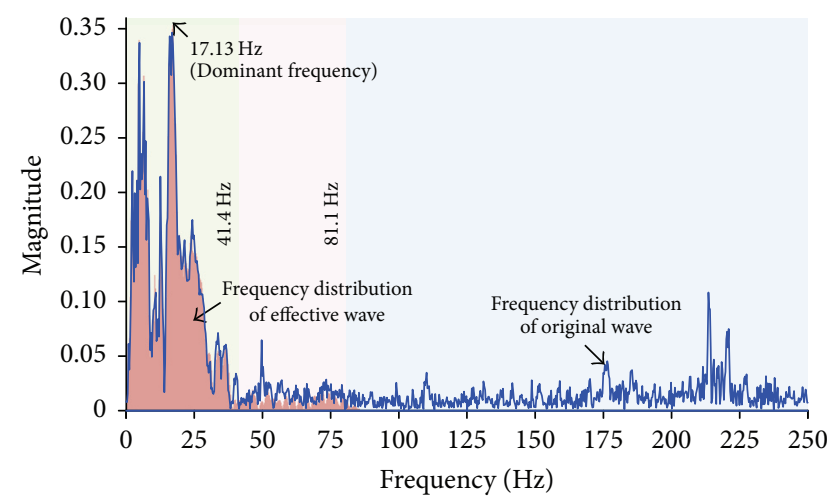

FIGURE 11: Comparison of the frequency spectrum of the extracted effective microseismic wave and the original measured wave.

The characteristics of the dominant parameters again clearly verify that the frequency bandwidth of the effective wave component becomes narrow and is concentrated in the low range gradually with the increase in the propagation distance. Wave energy decreases because of the absorption effect of the propagation medium. Despite the noise interference, higher values of the SNR and the energy-reservation ratio indicate that the microseismic wave component in the original signal is precisely identified and effectively extracted by using the scale-changed domain decomposition. In addition, the frequency separation effect is accordingly improved the most. Microseismic waves with long propagation distance are more desirable for the effective wave component extraction because of the increasing noise interference.

\section{Discussion}

5.1. Effect of the Scale-Changed Tri-Domain Decomposition on Noise Reduction. Precise identification and effective extraction of the microseismic wave component are fundamental and quite essential for the processing of the original measuring signal. Because of noise interference, most of the original measuring signals cannot be directly applied to the subsequent quantitative analysis, for example, the focus positioning and focus energy calculation. A denoising process targeting this type of high noise signal is indispensable. The approaches applied in noise reduction focused on signal denoising. Differences lie in the fundamental point, the processing mode, and the final effect. The purposes of previous methods are noise elimination from the original signal while retaining the effective wave component. However, the purpose of the scale-changed domain decomposition is effective wave component extraction from the original signal by eliminating the noise. The arrival time is one of the key parameters closely related to the focus positioning and the focus energy calculation. Any slight time error induces the decrease in the reliability of the arrival time. Domain decomposition plays a significant role in the correction of the arrival times of longitudinal and transverse waves. The arrival times of longitudinal and transverse waves identified 


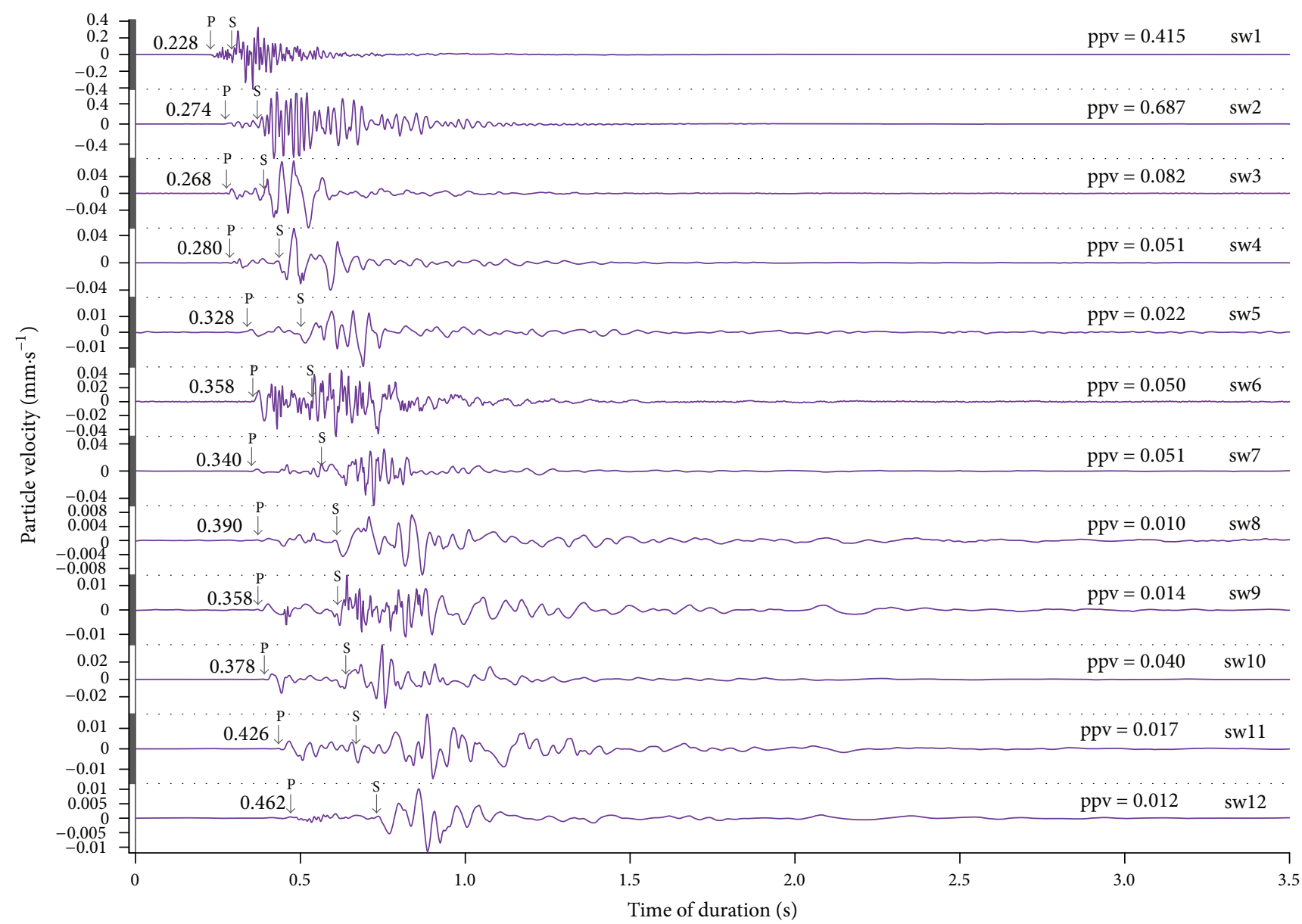

FIGURE 12: Recognizable waveform of the pure microseismic waves reconstructed via the extracted effective wave component in other monitoring channels.

from the purified extracted microseismic wave contribute to ensuring the reliability of focus repositioning and focus energy recalculation.

Noise applied in this case is only a regular reference for the original measuring signal. The reference noise helps to control the reliability of the effective microseismic wave. It is not the real noise in sw12. The reason why the reference noise is used in this case study is that the noise mixed in wave sw12 occurs repeatedly and has a high correlation coefficient with the reference noise. Because of the repeatability of the noise waveform, the reference noise is intercepted from the front portion of the long-duration measuring signal without any microseismic wave component. Using the reference noise, the noise form in the original sw12 can be determined. After the effective component extraction, the eliminated noise component from sw12 is compared with the reference noise to verify the denoising effect of this approach. For most of the original measuring waves, both real microseismic waves and mixed noise are completely unknown, which would bring difficulties into the threshold determination of the frequency and the wavelet coefficient.

5.2. Effect of the Frequency Threshold on the Identification and Extraction of the Microseismic Wave Component. When applying the scale-changed domain decomposition, precise identification of the thresholds corresponding to the wave frequency and wavelet coefficient in a specific time domain is key. The frequency threshold is applied to achieve the preliminary process of the original measured wave, and the wavelet coefficient threshold achieves advanced fine extraction of the effective wave component. The purpose of preliminary wave extraction using a frequency threshold is filtering the noise component in the high frequency range, by which the entire effective wave component is not impaired. For microseismic wave sw12, the frequency distribution in the entire time domain is relatively regular. Here, two threshold frequencies, $31.25 \mathrm{~Hz}$ and $81.1 \mathrm{~Hz}$, are adequate to preliminarily extract the effective wave component. Effective identification results of the microseismic wave component are shown in Figure 13(a) after the preliminary extraction using the frequency threshold, by which most of the high frequency redundant component is already rejected. For most of the original measuring waves, the frequency distribution in the time domain is quite irregular because of the interference of aperiodic nonquantitative environmental noise. It shall be better once the separate threshold frequency is confirmed at a time window as short as possible, and all thresholds are determined according to the total wave structure. If no frequency 


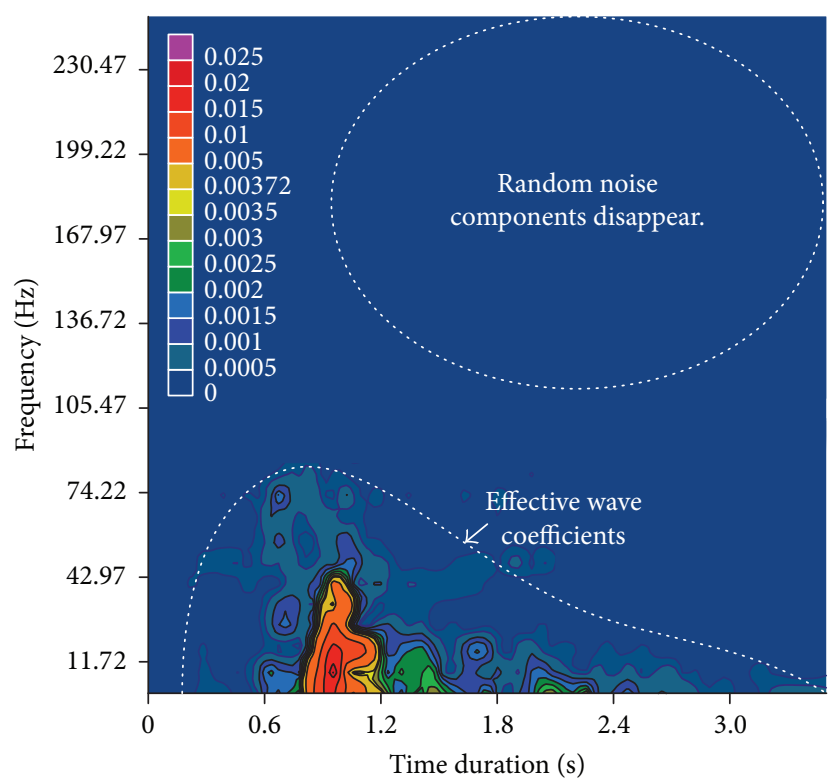

(a)

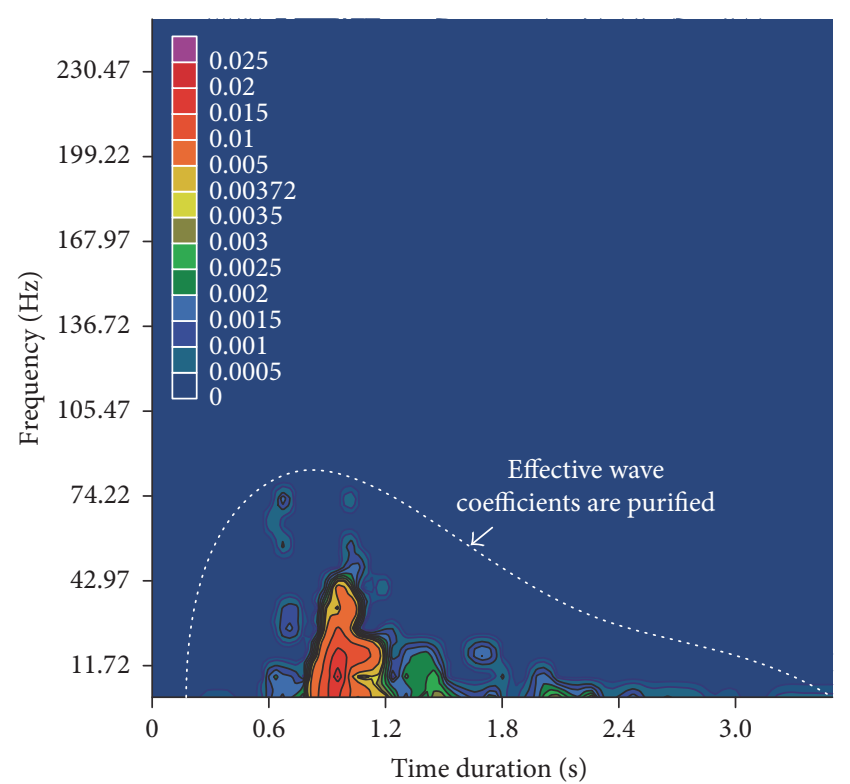

(b)

FIGURE 13: Identification results of the effective microseismic wave component after the preliminary extraction via the threshold frequency and advanced extraction via the threshold coefficient.

threshold is applied, then the effective wave component of sw12 can be extracted completely only because the wavelet coefficient threshold is greater than $3.72 \times 10^{-3}$, which is just a threshold coefficient applied for the conventional wavelet packet transform. Single wave component extraction is not sufficiently effective and undoubtedly decreases the reliability of the effective wave.

\subsection{Effect of the Wavelet Coefficient Threshold on the Iden-} tification and Extraction of the Microseismic Wave Component. Because the wavelet coefficient magnitude of the noise component in the high frequency range is obviously greater than that of the noise component in the low frequency range, the wavelet coefficient threshold used in low frequency can be reduced once the high frequency noise component is removed. In addition, the magnitude of the wavelet coefficient threshold set for extracting the effective wave component could be decreased; this approach plays an excellent role in protecting the details of the effective wave component. The feasibility of pure microseismic wave reconstruction using only wavelet coefficients of the effective wave component consequently increases. The greater wavelet coefficient threshold results in more general identification and extraction of the effective microseismic wave component. The extracted microseismic wave approaches reality only as the wavelet coefficient threshold decreases in a specific frequency domain. The more precise identification results of the microseismic wave component are shown in Figure 13(b) after the advanced extraction using the wavelet coefficient threshold.

Microseismic waves extracted only by the greater wavelet coefficient threshold shall be quite pure but unrealistic because other effective wave components whose coefficient magnitude is lower than the coefficient threshold are lost with the noise component. As a result, wave component extraction using the single threshold (frequency or wavelet coefficient) is defective in this case. Multistage synergistic interaction of the frequency threshold and the wavelet coefficient threshold in a specific time domain achieves a better denoising effect via the identification and extraction of the effective wave component and ensures the reliability of the extracted microseismic waves. The use of more thresholds ensures a better extracting process of the effective wave component, thus enabling the excellent analytical solution in the grid density of the triaxial domains among duration, frequency, and wavelet coefficient.

\section{Conclusions}

The following conclusions are made from the above results and discussion.

(1) Scale-changed tri-domain decomposition analysis is a comprehensive approach for fine wave processing. The effective microseismic wave component is identified and extracted as precisely as possible via the fine decomposition analysis on triaxial domains composed of time, frequency, and wavelet coefficients. Using the domain decomposition, the extracted wave component is fairly pure, thereby ensuring the reliability of the new wave reconstructed by the effective component.

(2) Accurate identification of the threshold frequency and the threshold coefficient is the key to the application. Interaction of the threshold frequency and the threshold coefficient in the time domain promotes the analytical solution of subwaves. The threshold frequency is determined by the spectral distribution of the original wave in the time domain; the threshold coefficient is determined by the peak wavelet coefficient of the redundant component in the frequency domain. More reasonable threshold frequencies 
and threshold coefficients achieve better extraction of the effective wave component.

(3) The precision of the scale-changed decomposition in the time domain, frequency domain, and wavelet coefficient domain is totally dependent upon the complexity of the original wave structure. The spectral characteristics of the real wave and the redundant noise component improve the precision recognition. The dominant parameters, such as the arrival time, dominant frequency, and wave energy, are all corrected and reserved for the extracted effective wave component via the scale-changed tri-domain decomposition. The corrected wave parameters contribute to the accurate repositioning of the focus and recalculation of the energy.

\section{Additional Points}

Highlights. (i) Domain decomposition analysis is an effective approach for precise wave processing. (ii) Accurate threshold identification of frequency and wavelet coefficient is a key. (iii) Wave spectral characteristics on triaxial domains promote the extracting precision. (iv) Effective wave component ensures the reliability of reconstructed microseismic wave.

\section{Conflicts of Interest}

The authors declare that there are no possible conflicts of interest in the manuscript.

\section{Authors' Contributions}

Shengdong Liu, Hideki Shimada, and Mingwei Zhang conceived, designed, and summarized the analytical method; Qingbin Meng and Mingwei Zhang performed the field experiments and obtained the data; Shengdong Liu, Qingbin Meng, Hideki Shimada, and Mingwei Zhang analyzed the data; Mingwei Zhang wrote the paper.

\section{Acknowledgments}

The financial and general support for this research provided by the Fundamental Research Funds for the Central Universities (no. 2015QNA62) and the National Key R\&D Program of China (no. 2016YFC0600900) is gratefully acknowledged. This fund covers the costs of publishing the work. In addition, the authors would like to express their sincere gratitude to the Xingcun Coal Mine for the support with the field experiments.

\section{References}

[1] M. Cai, P. K. Kaiser, and C. D. Martin, "Quantification of rock mass damage in underground excavations from microseismic event monitoring," International Journal of Rock Mechanics and Mining Sciences, vol. 38, no. 8, pp. 1135-1145, 2001.

[2] M. Ge, "Efficient mine microseismic monitoring," International Journal of Coal Geology, vol. 64, no. 1-2, pp. 44-56, 2005.

[3] K. J. Yol, Y. Kim, S. W. Kang, and H. S. Jang, "Development of microseismic monitoring and analysis system for prediction of ground subsidence," Journal of the Korean Society of Mineral and Energy Resources Engineers, vol. 48, no. 6, pp. 764-774, 2011.

[4] J.-P. Liu, X.-T. Feng, Y.-H. Li, S.-D. Xu, and Y. Sheng, "Studies on temporal and spatial variation of microseismic activities in a deep metal mine," International Journal of Rock Mechanics and Mining Sciences, vol. 60, pp. 171-179, 2013.

[5] I. V. Rodriguez and M. D. Sacchi, "Microseismic source imaging in a compressed domain," Geophysical Journal International, vol. 198, no. 2, pp. 1186-1198, 2014.

[6] N. W. Xu, F. Dai, Z. Z. Liang, Z. Zhou, C. Sha, and C. A. Tang, "The dynamic evaluation of rock slope stability considering the effects of microseismic damage," Rock Mechanics and Rock Engineering, vol. 47, no. 2, pp. 621-642, 2014.

[7] N.-W. Xu, C.-A. Tang, H. Li et al., "Excavation-induced microseismicity: microseismic monitoring and numerical simulation," Journal of Zhejiang University SCIENCE A, vol. 13, no. 6, pp. 445-460, 2012.

[8] D. A. Angus, A. Aljaafari, P. Usher, and J. P. Verdon, "Seismic waveforms and velocity model heterogeneity: Towards a fullwaveform microseismic location algorithm," Journal of Applied Geophysics, vol. 111, pp. 228-233, 2014.

[9] J. P. Verdon and J.-M. Kendall, "Detection of multiple fracture sets using observations of shear-wave splitting in microseismic data," Geophysical Prospecting, vol. 59, no. 4, pp. 593-608, 2011.

[10] M. Zhang, T. Sasaoka, H. Shimada, and K. Matsui, "Comparison of optimal basis function for the underground microseismic wave processing in wavelet packet transform," Memoirs of the Faculty of Engineering, Kyushu University, vol. 73, no. 3, pp. 7185, 2013.

[11] E. Baziw and I. Weir-Jones, "Application of Kalman filtering techniques for microseismic event detection," Pure and Applied Geophysics, vol. 159, no. 1-3, pp. 449-471, 2002.

[12] A. A. Spivak and S. B. Kishkina, "The use of microseismic background for the identification of active geotectonic structures and determination of geodynamic characteristics," Izvestiya, Physics of the Solid Earth, vol. 40, no. 7, pp. 573-586, 2004.

[13] J. B. Tary, R. H. Herrera, and M. van der Baan, “Time-Varying autoregressive model for spectral analysis of microseismic experiments and long-period volcanic events," Geophysical Journal International, vol. 196, no. 1, pp. 600-611, 2013.

[14] Z. Du, G. R. Foulger, and W. Mao, "Noise reduction for broadband, three-component seismograms using data-adaptive polarization filters," Geophysical Journal International, vol. 141, no. 3, pp. 820-828, 2000.

[15] F. Song, N. R. Warpinski, M. N. Toksöz, and H. S. Kuleli, "Full-waveform based microseismic event detection and signal enhancement: An application of the subspace approach," Geophysical Prospecting, vol. 62, no. 6, pp. 1406-1431, 2014.

[16] F. Forghani-Arani, M. Willis, S. S. Haines, M. Batzle, J. Behura, and M. Davidson, "An effective noise-suppression technique for surface microseismic data," Geophysics, vol. 78, no. 6, pp. KS85KS95, 2013.

[17] W. Peng, C. Xu, W. Yi-Bo, W. Lu-Chen, and Z. Hong-Yu, "Automatic event detection and event recovery in low SNR microseismic signals based on time-frequency sparseness," Chinese Journal of Geophysics, vol. 57, no. 5, pp. 739-749, 2014.

[18] A. C. To, J. R. Moore, and S. D. Glaser, "Wavelet denoising techniques with applications to experimental geophysical data," Signal Processing, vol. 89, no. 2, pp. 144-160, 2009. 
[19] I. Vera Rodriguez, D. Bonar, and M. Sacchi, "Microseismic data denoising using a $3 \mathrm{C}$ group sparsity constrained timefrequency transform," Geophysics, vol. 77, no. 2, pp. V21-V29, 2012.

[20] V. Grechka and S. Yaskevich, "Inversion of microseismic data for triclinic velocity models," Geophysical Prospecting, vol. 61, no. 6, pp. 1159-1170, 2013.

[21] A. Kushnir, A. Varypaev, I. Dricker, M. Rozhkov, and N. Rozhkov, "Passive surface microseismic monitoring as a statistical problem: Location of weak microseismic signals in the presence of strongly correlated noise," Geophysical Prospecting, vol. 62, no. 4, pp. 819-833, 2014.

[22] Y. Vaezi and M. Van der Baan, "Analysis of instrument self-noise and microseismic event detection using power spectral density estimates," Geophysical Journal International, vol. 197, no. 2, pp. 1076-1089, 2014.

[23] I. Daubechies, "The wavelet transform, time-frequency localization and signal analysis," Institute of Electrical and Electronics Engineers Transactions on Information Theory, vol. 36, no. 5, pp. 961-1005, 1990.

[24] R. C. Penner, "On Hilbert, Fourier, and wavelet transforms," Communications on Pure and Applied Mathematics, vol. 55, no. 6, pp. 772-814, 2002.

[25] P. J. Wood, "Wavelets and Hilbert modules," Journal of Fourier Analysis and Applications, vol. 10, no. 6, pp. 573-598, 2004.

[26] A. Graps, "An introduction to wavelets," IEEE Computational Science \& Engineering, vol. 2, no. 2, pp. 50-61, 1995.

[27] B. Walczak and D. L. Massart, "Noise suppression and spinal compression using the wavelet packet transform," Chemometrics and Intelligent Laboratory Systems, vol. 36, no. 2, pp. 81-94, 1997.

[28] R. X. Gao and R. Yan, Wavelet Packet Transform, Springer Science \& Business Media, Berlin, Germany, 2011.

[29] M. Misiti, Y. Misiti, G. Oppenheim, and J. M. Poggi, Wavelet Toolbox, The MathWorks Inc., Natick, Mass, USA, 1996.

[30] N.-R. Zhou and F. Jia, "Parseval theory of complex wavelet transform for wavelet family including rotational parameters," Communications in Theoretical Physics, vol. 50, no. 3, pp. 598602, 2008.

[31] L.-Y. Hu and H.-Y. Fan, "Inversion formula and Parseval theorem for complex continuous wavelet transforms studied by entangled state representation," Chinese Physics B, vol. 19, no. 7, pp. 263-267, 2010. 


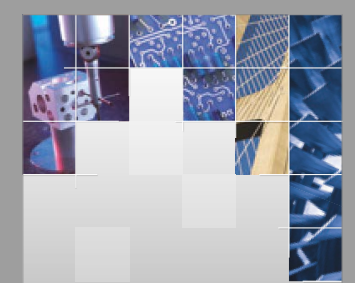

\section{Enfincering}
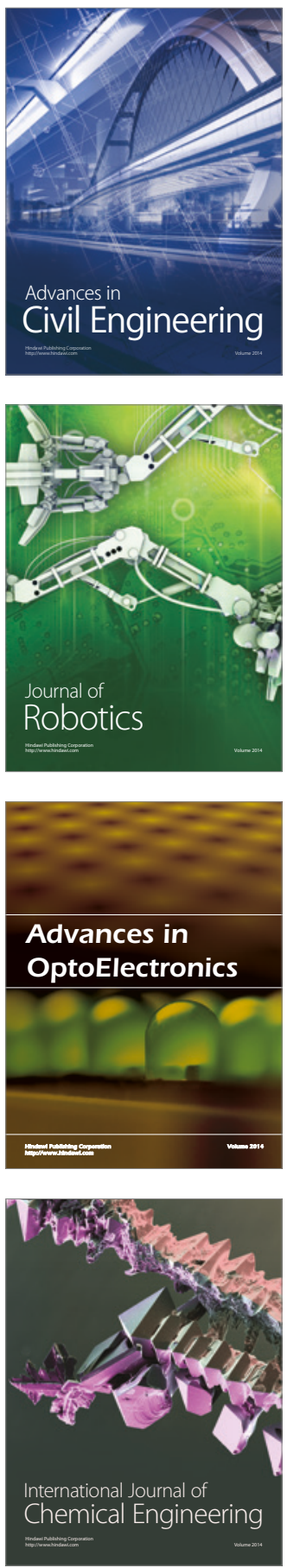

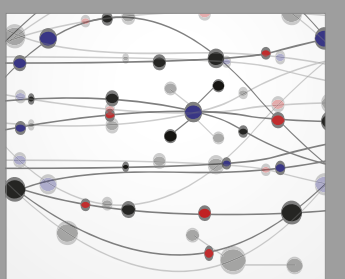

The Scientific World Journal

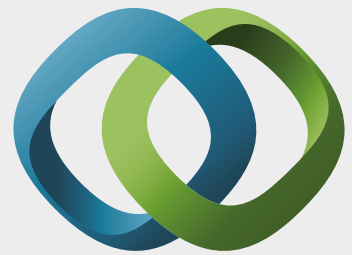

\section{Hindawi}

Submit your manuscripts at

https://www.hindawi.com
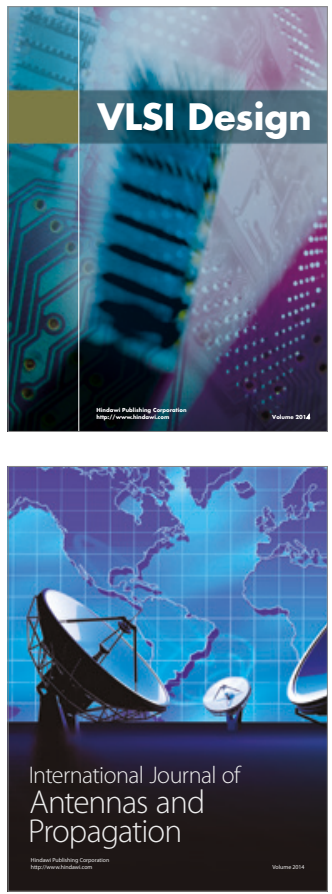

\section{Rotating}

Machinery
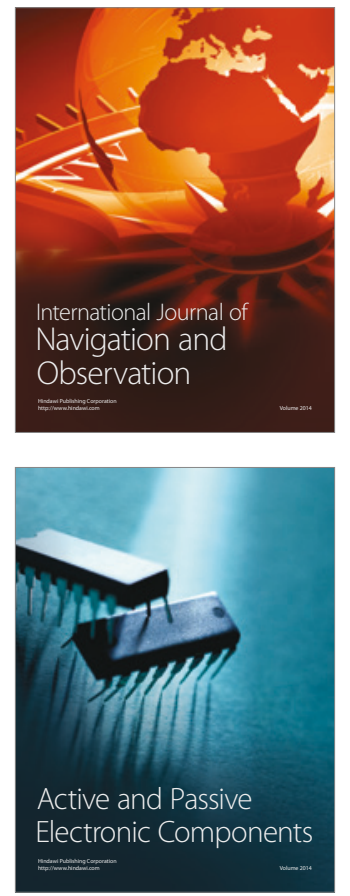
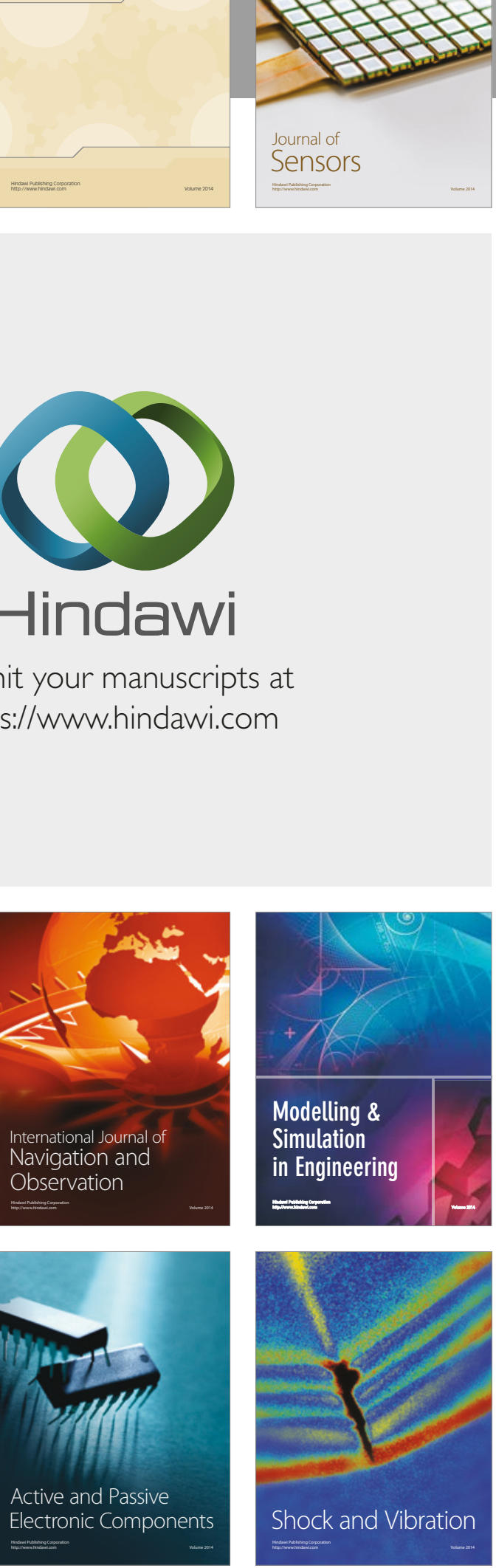
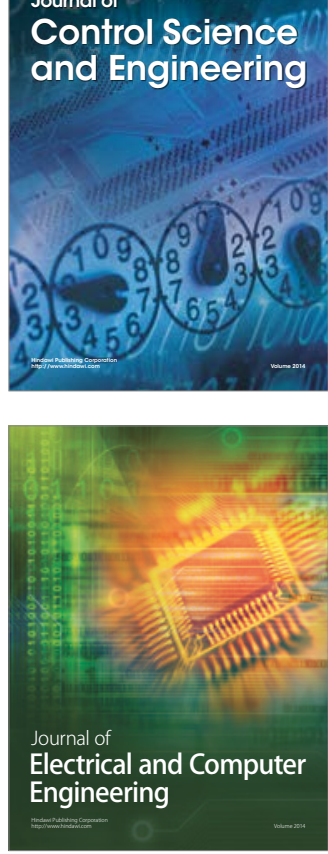

Distributed

Journal of

Control Science

and Engineering
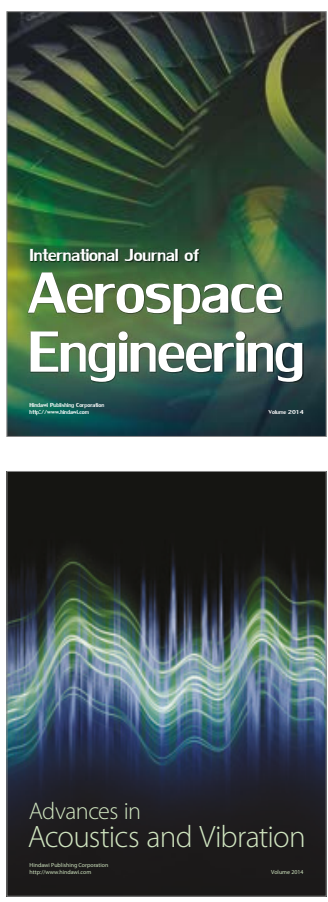

Sensor Networks 